\title{
La neige collante \\ Essai de cartographie du risque sur l'ensemble de la France
}

\author{
Wet-snow accretion on power lines \\ Cartographic examination of risks throughout France
}

\author{
V. Deneau et P. Guillot
}

E.D.F - D.T.G.

Plusieurs incidents graves, privant d'électricité des centaines de milliers d'usagers pendant plusieurs jours, ont endommagé les réseaux de transport et de distribution notamment dans le quart Sud-Est de la France au cours de la dernière décennie.

Au cours des années 80 , ils ont affecté :

- le Languedoc-Roussillon, 11 janvier 1981 [33, 34, 35];

- Saint-Etienne et sa région, 26 novembre 1982 [38, 39].

Ces avaries surviennent lors de chutes intenses de neige mouillée, qui forme autour des câbles des manchons dont le poids peut atteindre 5 voire $10 \mathrm{~kg} / \mathrm{m}$. Dans un premier stade, cette surcharge mécanique provoque des déclenchements, par suite des différences d'allongement (amorçage entre phases et câble de garde), puis, dans les cas plus graves, entraîne la déformation puis la rupture des supports qui sont calculés, selon les normes françaises actuelles, pour une surcharge de $2 \mathrm{~cm}$, plus rarement $4 \mathrm{~cm}$, de " givre de densité 0,6 ", soit environ 2 et $4 \mathrm{~kg} / \mathrm{m}$.

En réalité, la neige collante est un phénomène complètement différent du givre et du verglas : ceux-ci adhèrent par la congélation de l'eau surfondue; au contraire, la neige collante, ainsi que l'ont montré les études japonaises, tient au câble par la tension capillaire de l'eau liquide qu'elle contient, la température de l'air ambiant restant positive (1).

En France, à la demande des Centres régionaux de transport, la D.T.G. a été amenée depuis 1973 :

(1) Les nombreuses publications ( 1 à 10) de " Environnement Canada " à Toronto, l'hydro-Québec, l'Université de Chicoutimi, le CRREL, le Laboratoire du Puy-de-Dôme traitent toutes de l'accrétion de givre et de verglas (sauf l'article de Bauer [3] qui est le seul à décrire un cas de neige collante dans le Saskatchewan en avril 1971). L'étude de Colbeck dans [10] traite bien des forces capillaires dans la neige mouillée, mais relate essentiellement les expériences japonaises.
- à étudier la possibilité de prévision en temps réel d'après les conditions synoptiques [23, 37];

- à dresser, sur les zones des C.R.T.T. de Marseille et Toulouse, une première version de carte du risque d'après les données climatologiques $[27,28,30,31]$.

Le but de la présente note est de proposer des simplifications permettant d'étendre rapidement l'esquisse de cette carte à l'ensemble de la France.

$\mathrm{Au}$ préalable, il nous paraît utile de résumer les recherches de nos collègues au Japon, qui est le premier pays, où le problème de la neige collante ait été identifié et fait l'objet de contre-mesures appropriées. Une coopération franco-japonaise sur ce thème a été décidée après un échange de mission en 1981 et 1982.

\section{1 - Les études japonaises}

Les chercheurs japonais ont identifié successivement deux catégories extrêmes de neige collante :

1) La neige dite "sèche ", contenant en réalité juste assez d'eau liquide pour former sur les fils un dépôt cohérent de densité inférieure à 0,2 , à une température de l'air comprise entre 0 et $1{ }^{\circ} \mathrm{C}$, et par vent faible de vitesse inférieure à $2-3 \mathrm{~m} / \mathrm{s}$; en cas de vent plus fort, le dépôt relativement fragile se détache. On l'observe couramment par type de temps dit "de Mousson ", c'est-à-dire dans le flux de secteur ouest qui apporte des précipitations régulièrement abondantes sur les côtes ouest du Japon. Les mécanismes d'adhérence et de croissance de ce type de dépôt ont été méticuleusement décrits par le Docteur Shoda (1953), alors qu'il dirigeait le centre d'études des avalanches des chemins de fer japonais à Shiozawa, 


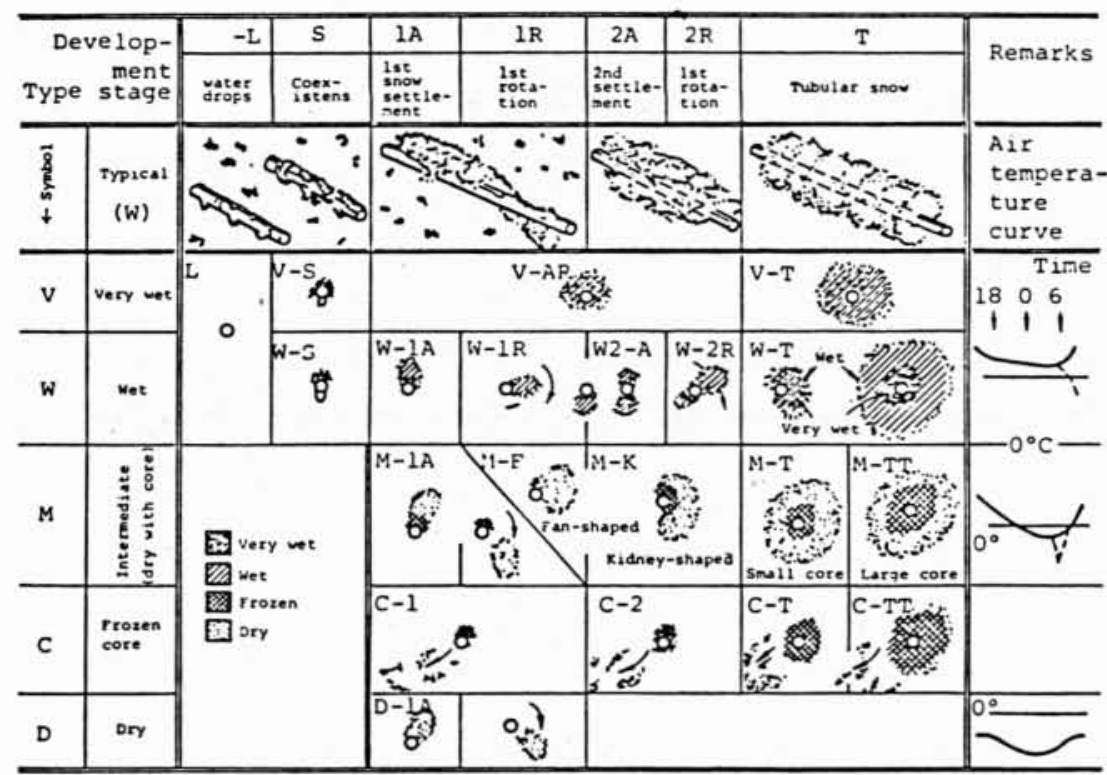

Tableau I

Classification établie par

M. Shoda district de Niigata. Vu leur densité relativement faible, et leur fragilité, le poids de ces manchons n'atteint qu'assez rarement une valeur dangereuse pour les lignes.

Shoda n'avait pas observé de densité du manchon supérieure à 0,2 , ni de surcharge supérieure à $2 \mathrm{~kg} / \mathrm{m}$; il résumait ses observations en un tableau reproduit sur le tableau $I$.

Il avait donc bien observé le processus d'enroulement du manchon, mais il avait ignoré la possibilité d'accrochage et de tassement de la neige sur le cable, par vent fort, jusqu'à une densité très supérieure à celle déposée au sol.

2) La neige "mouillée ", par vent fort de 10 et même $20 \mathrm{~m} / \mathrm{s}$, et une température de l'air de 1 à $3^{\circ} \mathrm{C}$, forme des manchons très lourds, saturés d'eau liquide, de densité 0,6 à 0,8 , qui s'enroulent autour du câble en s'égouttant de leur surplus d'eau. Ce type de temps dit " de dépression " correspond au passage de profondes dépressions mobiles, alimentées en air chaud en altitude, sur le nord de Honshu et l'île d'Hokkaido. Ce phénomène, plus rare mais plus violent que la neige de "Mousson ", a été la cause des avaries de lignes les plus graves survenues dans l'île d'Hokkaido. C'est pour le combattre que les ingénieurs de la Compagnie d'Electricité d'Hokkaido, Nakano, Goto, Higuchi (CIGRE 1974) ont eu l'idée, dès 1970, des bagues fixées sur les câbles, qui font obstacle à l'enroulement du manchon le long des spires hélicoïdales, et amorcent son décollement. Les bagues espacées de deux pas de toronnage $(30$ à $50 \mathrm{~cm})$ doivent être complétées par des contre-poids, tous les 50 à $100 \mathrm{~m}$, qui empêchent la rotation du câble par torsion élastique. Sans ces contrepoids, les bagues sont inefficaces en milieu de portée.

Au cours des années 1970, la Compagnie d'Electricité d'Hokkaido, le fabricant de câbles Furukawa EPC, et finalement l'institut des basses températures de Sapporo, ont monté plasieurs expériences pour reproduire, par projection de neige mouillée, le processus de formation des manchons : les électriciens ont pu ainsi tester l'efficacité des bagues, et des contre-poids anti-torsion; efficacité confirmée ensuite par des comparaisons en exploitation de lignes baguées et non baguées. Les physiciens ont mesuré l'adhérence capillaire de la neige mouillée sur les différents matériaux, filmé l'impact des flocons de neige, et constaté que, par vent fort, les pertes par rebondissement et arrachement sont telles que le coefficient d'accrétion $\alpha$ (voir ci-dessous) ne dépasse pas $20 \%$ [Wakahama, 1979, 15].

Les essais réalisés à Sapporo, de projection en soufflerie de neige fraîche, ramassée au sol, passée au tamis et mouillée par de l'eau pulvérisée, simulaient le cas extrême du type "dépression " avec vent fort. Une grande partie des cas réels observés au Japon - et la quasitotalité de ceux qui ont été observés en France - sont en fait des cas intermédiaires, où les vents sont modérés $(5 \mathrm{~m} / \mathrm{s})$ voire quasi nuls (Saint-Etienne), donnant une densité moyenne du manchon apparemment comprise entre 0,3 et 0,5 .

A cet égard, les essais réalisés à Nikko par la Compagnie Furukawa, en déchargeant simplement la neige dans une trémie à la verticale au-dessus des câbles, sont sans doute plus représentatifs de nos cas les plus fréquents.

A partir de 1974, après le décès de Shoda, Y. Sakamoto et Y. Hirayama, au C.R.I.E.P.I. (Centre de recherche de la fédération des 9 compagnies d'électricité japonaise), ont repris les essais d'une équation générale de calcul du poids du manchon, applicable aussi bien au type "mousson ", qu'au type "dépression " et aux cas intermédiaires [19, 20]. 
En partant de l'accrétion différentielle :

$$
2 \pi R \rho_{i} d R=\alpha p_{n} \cdot 2 R \cdot d t
$$

où $t$ est le temps

$R$ : le rayon du manchon

$\rho_{i}$ : la densité de la neige déposée sur le conducteur

$p_{n}$ : précipitation normale au câble, par unité de temps,

$p_{n}=p \sqrt{1+\left(\frac{V \sin \theta}{V s}\right)^{2}}:$

$p$ : précipitation verticale

$V$ : vitesse du vent

$\theta$ : angle du vent et du câble

Vs : vitesse de chute des flocons.

$\alpha$ : le taux d'accrétion, c'est-à-dire la fraction de $p_{n}$ qui reste fixée au manchon

$P_{n}=\int_{0}^{\top} p n d t$

après intégration :

$$
R-R_{0}=\frac{1}{\pi} \frac{\alpha}{\rho i} P_{n}
$$

$R_{0}$ : rayon du conducteur

W : masse de neige, par unité de longueur du câble :

$$
W=\pi \rho i\left(R^{2}-R^{2}{ }_{0}\right)
$$

ou encore d'après (2) :

$$
W=\pi \rho i\left[\left(\frac{\alpha P_{n}}{\pi \rho i}+R_{0}\right)^{2}-R_{0}^{2}\right]
$$

Voici quelques essais d'application à des cas connus au Japon et en France :

\begin{tabular}{|l|c|c|c|c|c|c|}
\hline \multicolumn{1}{|c|}{ Lieu et date } & $\begin{array}{c}\text { Vsin } \theta \\
\mathrm{m} / \mathrm{s}\end{array}$ & $\begin{array}{c}\mathrm{P} \\
\mathrm{mm}\end{array}$ & $\begin{array}{c}\mathrm{2R} \\
\mathrm{cm}\end{array}$ & $\begin{array}{c}\mathrm{W} \\
\mathrm{kg} / \mathrm{m}\end{array}$ & $\alpha$ & $\begin{array}{c}\rho^{\prime} \\
\mathrm{g} / \mathrm{cm}^{3}\end{array}$ \\
\hline $\begin{array}{l}\text { Type " Mousson " } \\
\text { décrit par Shoda }\end{array}$ & 0 & 20 & 13 & 1,3 & 1 & 0,1 \\
\hline $\begin{array}{l}\text { Saint-Etienne } \\
26 \text { novembre 1982 }\end{array}$ & 0 & 60 & 13 & 4 & 1 & 0,3 \\
\hline $\begin{array}{l}\text { Toyama } \\
\text { 3 janvier 1981 }\end{array}$ & 4 & 50 & 14 & 4,6 & 0,33 & 0,3 \\
\hline $\begin{array}{l}\text { Perpignan } \\
11 \text { janvier 1981 }\end{array}$ & 5 & 40 & 16 & 9 & 0,55 & 0,45 \\
\hline $\begin{array}{l}\text { Bollène } \\
\text { 3 mars 1974 }\end{array}$ & 7 & 50 & 15 & 10 & 0,40 & 0,60 \\
\hline $\begin{array}{l}\text { Sendai } \\
24 \text { décembre 1980 }\end{array}$ & 8 & 70 & 16 & 8 & 0,16 & 0,40 \\
\hline $\begin{array}{l}\text { Cherbourg } \\
13 \text { février 1970 }\end{array}$ & 10 & 25 & 8 & 4 & 0,40 & 0,80 \\
\hline $\begin{array}{l}\text { Wakanai } \\
1^{\text {er décembre 1972 }}\end{array}$ & 12 & 40 & 14 & 10,6 & 0,29 & 0,70 \\
\hline
\end{tabular}

Les chiffres ci-dessus ne sont évidemment que des estimations plausibles, et les valeurs de $\alpha$ sont choisies pour convenir.

En fait, les paramètres météorologiques $p, V, \theta$, ne sont pas mesurés sur le site même, et fluctuent largement au cours de l'épisode. Les "mesures " de $R, W$ ou plutôt leurs estimations après coup d'après les avaries, ne permettent donc de déduire dans chaque cas qu'une valeur moyenne $\bar{\alpha}$, qui intègre les effets de non-linéarités ( $W$ croît comme $\alpha^{2} P_{n}^{2}$ ), des seuils mal connus (en règle générale le début de la précipitation est liquide), voire de sous-estimation des valeurs locales de $p$ et $V$. Les japonais ont trouvé dans certains cas $\alpha$ supérieur à 1 .

Le C.R.I.E.P.I a néanmoins tenté, en utilisant son fichier d'incidents, et les estimations correspondantes de $R$ et $W$, d'ajuster des équations de régression donnant $\alpha$ et $\rho$ en fonction des variables climatologiques (température, vitesse et direction du vent) mesurées aux stations les plus proches (et supposées représentatives...) au lieu de l'incident.

Par exemple, il obtient la double équation suivante pour le type "dépression" :

$$
\begin{aligned}
& \alpha=e^{-17}\left(T / T_{50}-0,45\right)^{2}-0,039 \mathrm{~V} \sin : \theta \\
& -0,0075\left(2 R_{0}\right)+0,26 \\
& \quad(T \text { température en dessous de laquelle } 50 \% \text { des cas de } \\
& \quad \text { précipitation sont en neige }) \\
& \rho i=0,33+0,3 T+0,007 \mathrm{~V} \sin \theta
\end{aligned}
$$

Cette double équation, ajustée sur 14 cas d'incidents type dépression, aboutit pour $\alpha$ à une valeur qui paraît encore beaucoup trop forte, démentie à la fois par le cas de Sendai $(\alpha=0,16)$ et la constatation en Laboratoire de Wakahama $(\alpha<0,2)$. Faute d'une connaissance expérimentale suffisamment solide de $\alpha$, et $W$ variant comme $\alpha^{2} / \rho$, l'ajustement de régressions donnant la surcharge à partir des données climatologiques, paraît pour le moment inapplicable. Nous ignorons la représentativité des observations au Japon, mais en France elle est évidemment insuffisante.

On sait seulement que $\rho i$ est une fonction croissante de la température $T$ et de la vitesse du vent $V$, et que $\alpha$, optimum entre 1 et $2{ }^{\circ} \mathrm{C}$, et par vent nul, décroît rapidement avec $V$.

Le C.R.I.E.P.I. a donc repris, au cours de l'hiver 83-84 à Ishi-Uchi, des simulations en soufflerie, en chauffant le jet d'air, pour tenter de reproduire les cas intermédiaires donnant des intensités de manchon dans la gamme 0,3-0,5. Deux chercheurs français ont participé à ces essais en février 84 , et ont obtenu des manchons adhérant si étroitement aux câbles que l'efficacité des bagues parait nulle. L'analyse de ces résultats est en cours et là aussi se pose la question de la représentativité des conditions thermodynamiques de ces expériences (humidité de l'air chauffé, teneur en eau liquide de la neige ?...) par rapport aux cas réels observés en France. 


\section{2 - La neige collante en France}

Si l'on excepte le cas du Cotentin $(\rho>0,8$, vents de 10 à $15 \mathrm{~m} / \mathrm{s}$ ), les estimations les plus crédibles, sur les incidents récents, situent la densité entre .3 et .5 , vitesse du vent comprise selon les cas entre 0 et $8 \mathrm{~m} / \mathrm{s}$.

On remarque également que le vent s'inverse assez rapidement avec l'altitude, de secteur Nord venant de l'intérieur dans les basses couches, de sud-est amenant l'air humide au-dessus de quelques centaines de mètres. Ceci souligne les particularités de la structure verticale de l'air dans les basses couches, lors des chutes de neige collante :

- inversion de température en dessous de $500 \mathrm{~m}$ dans le cas du Languedoc;

- isothermie voisine de $0^{\circ}$ sur plus de $1500 \mathrm{~m}$ à SaintEtienne.
Particularités qui peuvent être à la fois une cause, par l'appel d'air froid dans les basses couches, qui accentue la vigueur du soulèvement de l'air humide, et une conséquence de l'intensité des précipitations, qui ne trouvent pas assez de calories pour fondre entièrement avant d'atteindre le sol.

Dans nos premières études, nous avions adopté comme indicateur des risques de neige collante $(\mathrm{PV})^{3 / 2}(\mathrm{P}=$ pluie en 6 heures, $\mathrm{V}=$ vent en $\mathrm{m} / \mathrm{s}$ pendant la précipitation). Saint-Etienne, où le vent était quasi nul, nous a montré qu'une accrétion importante peut se produire même sans vent; l'exposant ${ }^{3 / 2}$ est sans doute encore surestimé, l'expérience montrant que le poids du manchon croit heureusement beaucoup moins vite que $V^{2}$ et même que $V^{3 / 2}$.

Une équation de régression, mieux adaptée serait de la forme :

$W=k p^{\beta}\left(1+V^{2} \sin ^{2} \theta\right)^{\gamma}$, avec $\beta<1,5, \gamma<0,5$.

Les coefficients $\gamma$ et $\beta$ dépendant de la température, et de l'altitude.
Figure 1 - Hiérarchie des incidents.

Pour $W=0,01 P^{a(t)}(1+V \sin$ $\theta)^{1-(2 / 1000)}$

$\alpha$ de 1,2 à 1,5 pour $t$ de -1 à +3

$(1-z / 1000)$ de 1 à 0,5 pour $z$ de 0 à $500 \mathrm{~m}$, constant au-delà de $500 \mathrm{~m}$.

$\theta$ : angle du vent et de la ligne.

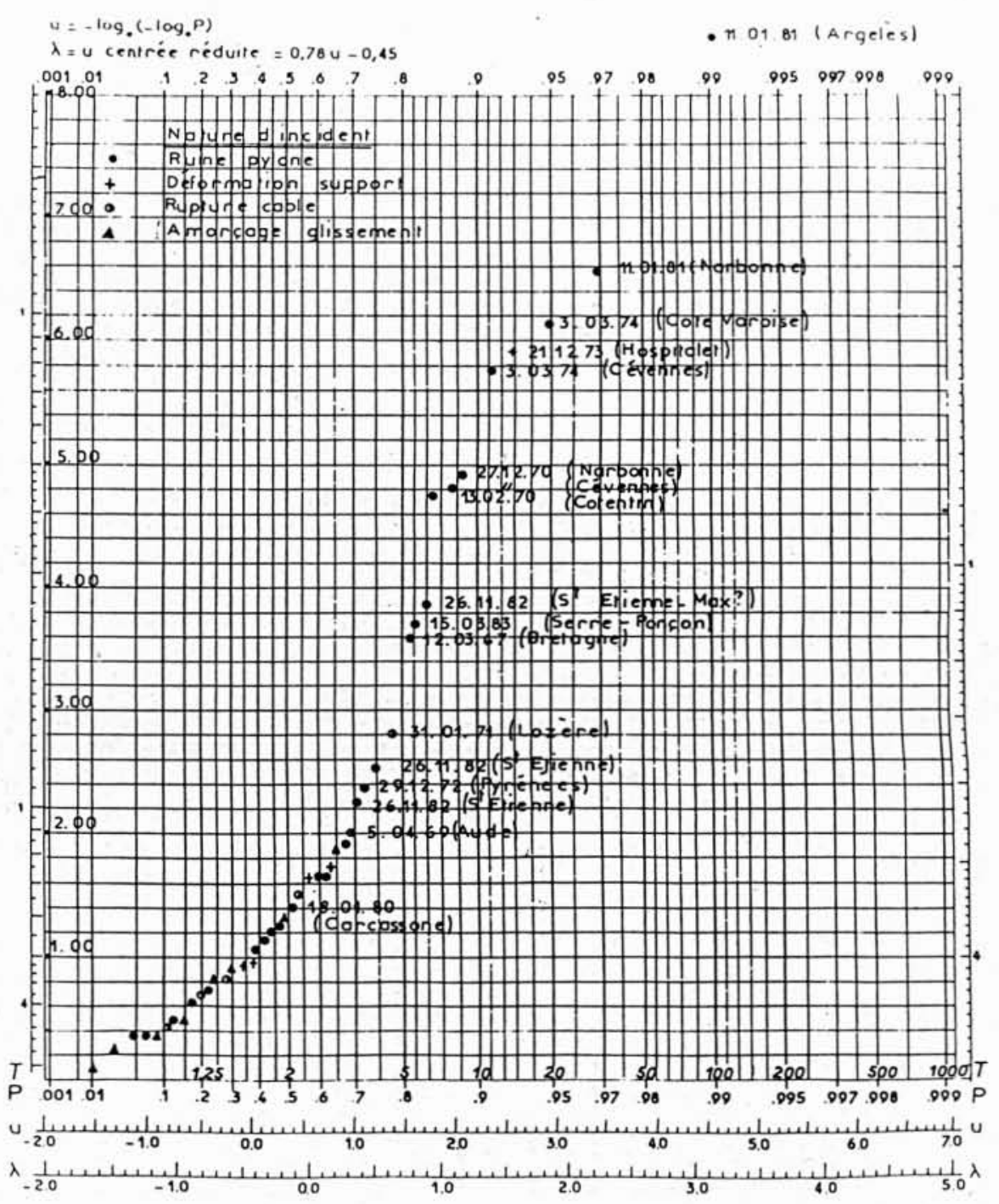


Des essais, en ce sens, ont été tentés, mais la taille réduite de l'échantillon de "mesures" relatives aux manchons ne permet pas d'en tirer des résultats significatifs [40]. (voir figure l).

Il apparaît en tous cas, qu'en plus d'une température légèrement positive le facteur essentiel pour la formation de neige collante est l'intensité de la précipitation. C'est une donnée climatologique assez bien connue, commodément extrapolable par son gradex, et qui doit permettre en première approximation une cartographie des risques relatifs.

Quant au rôle du vent, dans l'accrétion, sans le nier, il nous parait difficile actuellement de le quantifier, puisqu'il semble qu'au-delà de $2 \mathrm{~m} / \mathrm{s}$ il a deux effets opposés : accroissement du débit des flocons et diminution du coefficient d'accrétion par rebondissement et arrachement. Comme il accroît en outre la densité, son influence totale sur $W$ est peut être négligeable au-delà d'un seuil de l'ordre de 3 ou $5 \mathrm{~m} / \mathrm{s}$. (Sa prise en compte restera toutefois indispensable pour le projecteur, son incidence mécanique étant maximale lorsqu'il s'exerce perpendiculairement à la ligne).

\subsection{Le fichier d'incidents français}

Le fichier d'incidents (neige, givre, verglas) a été établi à partir d'informations collectées dans des services régionaux appliquant des consignes non homogènes. Outre son hétérogénéité, il présente l'inconvénient de ne prendre en compte que les cas où la défaillance de fourniture d'énergie a été notée; le nombre d'incidents semble s'accroître avec les années : plus grande rigueur dans les compte-rendus d'incidents allant de pair avec une plus grande exigence de la clientèle, accroissement de la densité du réseau ?...

Même en cas d'incidents graves, les témoignages sur les caractéristiques physiques des manchons, diamètre, densité, structure sont extrêmement rares.

Quoiqu'il en soit, les incidents importants sont tous répertoriés, et leur étude nous a permis de préciser les circonstances synoptiques de ces épisodes.

\subsection{Le fichier de neige collante}

Ce fichier a été établi, en éliminant du fichier d'incidents, les cas de givre et verglas, grâce à l'étude des situations météorologiques.

En effet le givre est généralement associé à des conditions anticycloniques, la neige collante est liée à des zones de basses pressions. Une étude interne faite en 1982, montrant que sur un échantillon de 71 cas, 68 avaient intéressé des régions allant des Pyrénées au Sud des Alpes.

Si l'on réduit l'échantillon, au cas où plusieurs lignes étaient affectées, cette proportion est ramenée à 31 sur 33 .

On voit donc que dans la quasi totalié des cas connus en France, la neige collante se limite au quart sud-est du pays, c'est-a-dire une vingtaine de départements des régions Provence Côte d'Azur, Languedoc Roussillon, Midi-Pyrénées, Rhône Alpes et Auvergne.

\subsection{Etude des incidents majeurs}

Les figures 2 à 7 représentent des conditions météorologiques liées aux "incidents neige" les plus marquants depuis 1970.

Si l'on excepte le cas du Cotentin, on note pour tous les autres, l'existence d'une zone dépressionnaire en Méditerranée.

Les intensités en 6 heures des précipitations dépassent $20 \mathrm{~mm}$ sauf pour le Cotentin $(16 \mathrm{~mm} / 6 \mathrm{~h})$. Les vents généralement faibles dans la plupart des cas dépassent $10 \mathrm{~m} / \mathrm{s}$ pour le Cotentin.

On notera également qu'il s'écoule en général environ 4 à 6 heures entre le début de la chute de neige et les premiers déclenchements par avarie.

On remarque donc que le cas du Cotentin s'oppose à tous les autres, y compris celui du 15 mars 1983 qui est un cas "d'altitude ". En particulier, on constatera que le vent dans les basses couches vient de "l'intérieur " (température $\simeq 0^{\circ}$ ), soulevant l'air méditerranéen plus chaud (température de la mer $\simeq 12^{\circ}$ ).

Dans le cas du Contentin, il s'agit d'un vent cyclonique sans contraste de masses d'air très marqué, du moins au sol.

On notera aussi que dans la plupart des incidents graves des manifestations orageuses sont observées, qui sont exceptionnelles en hiver en France sauf au moment des précipitations les plus intenses. Les éclairs étaient quasi-permanents pendant la chute de neige du 26 novembre 1982, autour de Saint-Etienne.

\subsection{Les deux types de neige collante}

De l'étude des " cas " comme de l'analyse de l'échantillon, il ressort que, comme au Japon, il existe en France deux types de temps à " neige collante ".

Un type "dépressionnaire ", le cas du Cotentin est pratiquement unique. Il peut sans doute arriver n'importe où, mais avec une probabilité extrêmement faible.

Un type "méditerranéen ", beaucoup plus habituel qui intéresse essentiellement le sud-est de la France.

$\mathrm{Si}$ le premier ressemble au cas "dépressionnaire" japonais, le second diffère sensiblement du type "mousson ", en raison de facteurs locaux très différents.

Il semble que la localisation de ce deuxième type en France puisse être associé à la présence proche d'air froid sur les reliefs (Massif-Central, Alpes) et d'une source chaude, la Méditerranée, la présence d'une dépression en Méditerranée déclenchant la "réaction". On notera que ce type de temps est très proche de celui qui engendre les pluies méditerranéennes d'automne; seules les conditions thermiques sont différentes.

La densité des manchons dans le type «méditerranéen " est aussi nettement plus forte; comprise entre 0,3 et 0,5 , c'est-à-dire au-dessus du seuil 0,2 indiqué par Shoda pour le type de "mousson".

\subsection{Efficacité des bagues en fonction du type de neige}

Les bagues sont d'autant plus efficaces que la neige est mouillée et qu'elle glisse facilement sur un film liquide autour du câble, à condition bien sûr que celui-ci soit lui-même empêché de tourner par les contre-poids. 


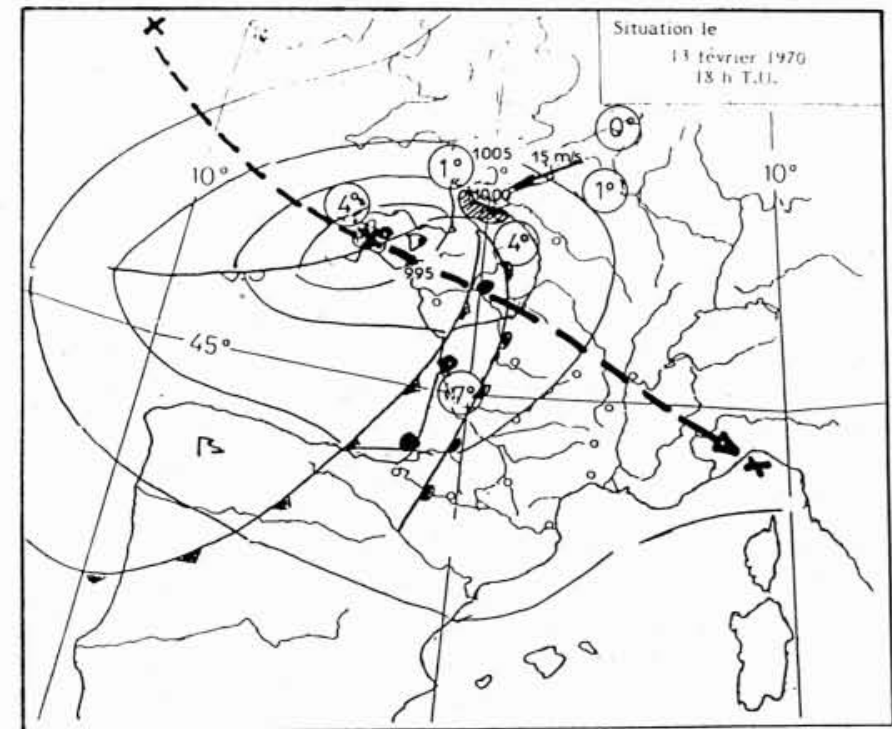

Avaries - 3 lignes $90 \mathrm{kV}$ Cotentin (Transport) Atitude: $<50 \mathrm{~m}$ Observations S/G. "Caen": "manchons de givre sur les câbles, $8 \mathrm{~cm}$
augmentation du Dpar neige collante". $\rightarrow$ de figece Observations météorologiques à Cherbourg helerogene

\begin{tabular}{|c|c|c|c|c|c|c|c|c|c|c|c|c|}
\hline \multicolumn{2}{|c|}{ Heures } & 00 & 03 & 06 & 09 & 12 & 15 & 18 & 21 & 00 & 03 & 06 \\
\hline \multicolumn{2}{|c|}{ Temperatures } & 2.4 & 1.7 & .1 & .9 & 3.1 & .9 & .7 & .1 & .2 & .2 & 0 \\
\hline \multirow{2}{*}{$\begin{array}{l}\vec{c} \\
\stackrel{y}{\infty}\end{array}$} & Direction & 30 & 30 & $\theta / 30$ & 14 & 10 & 08 & 06 & 06 & 04 & 02 & 02 \\
\hline & Force $\mathrm{m} / \mathrm{s}$ & 7 & 4 & 0 & 3 & 8 & 9 & 12 & 9 & 10 & 10 & 11 \\
\hline \multicolumn{2}{|c|}{ Precipitations $\mathrm{mm}$} & & & 3 & & & & 4 & & & & 21 \\
\hline \multicolumn{2}{|r|}{ ronologie } & & & & & $\frac{120}{120}$ & Bम0 & & & & & 3 \\
\hline
\end{tabular}

Figure 2

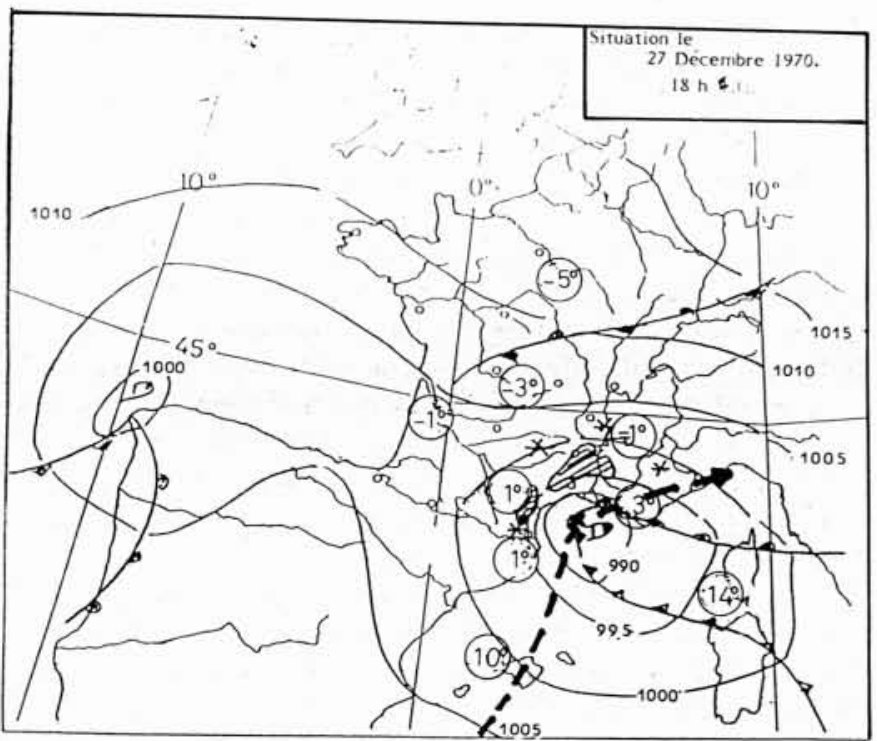

Avaries - Transports $3 \times 225 \mathrm{kV}-2 \times 150 \mathrm{kV}-18 \times 63 \mathrm{kV}$ Distribution : C.D. Montélimar - Ales - Le Puy - Nimes - Carcassonne
Altitude : 0 à $400 \mathrm{~m}$

Observations: manchons 10 à $15 \mathrm{~cm}$ (d'après photo - rapport S/G. Nimes)

Observations météorologiques à Nîmes

\begin{tabular}{|c|c|c|c|c|c|c|c|c|c|c|c|c|c|c|}
\hline \multicolumn{2}{|c|}{ 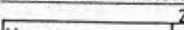 } & \multicolumn{4}{|c|}{26} & 27 & & & & & & & & 28 \\
\hline \multirow{2}{*}{\multicolumn{2}{|c|}{ Températures }} & 12 & 15 & 18 & 21 & 00 & 03 & 06 & 09 & 12 & 15 & 18 & 21 & 00 \\
\hline & & 3.6 & 3.3 & 1.5 & .9 & .8 & 1.7 & 2.2 & 2.0 & 2.3 & .9 & .9 & 1.3 & \\
\hline \multirow{2}{*}{ 茝 } & Direction & 02 & 04 & 02 & 02 & 36 & 36 & 02 & 36 & 36 & 01 & 36 & 34 & \\
\hline & Force $\mathrm{m} / \mathrm{s}$ & 5 & 5 & 5 & 2 & 4 & 2 & 5 & 5 & 6 & 9 & 12 & 8 & \\
\hline \multicolumn{2}{|c|}{ Précipitations ${ }_{\mathrm{mm}}$} & & & .6 & & & & 4. & & & & 21.1 & & 3.7 \\
\hline \multicolumn{2}{|c|}{ Chirunologie } & & 160 & & 60 & & & a 150 & 1000 & & 10 & $x$ & & \\
\hline
\end{tabular}

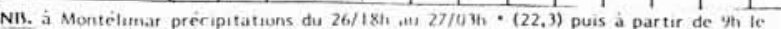

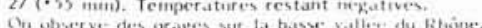

Figure 3

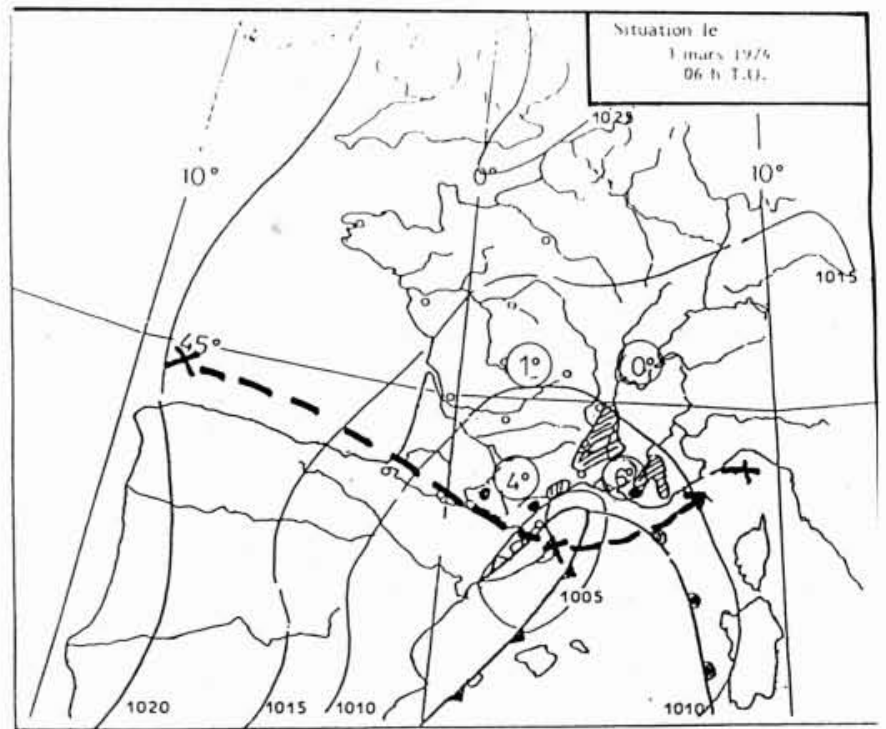

Avaries - Transport : $2 \times 400 \mathrm{kV}-8 \times 225 \mathrm{kV}-23 \times 63 \mathrm{kV}$ Distribution : reseau val
Altitude : $100-750 \mathrm{~m}$

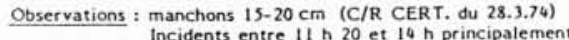

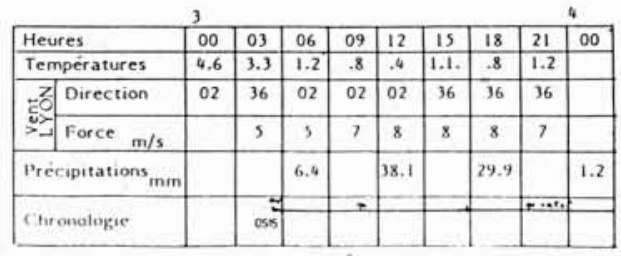

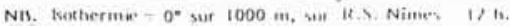

Figure 4

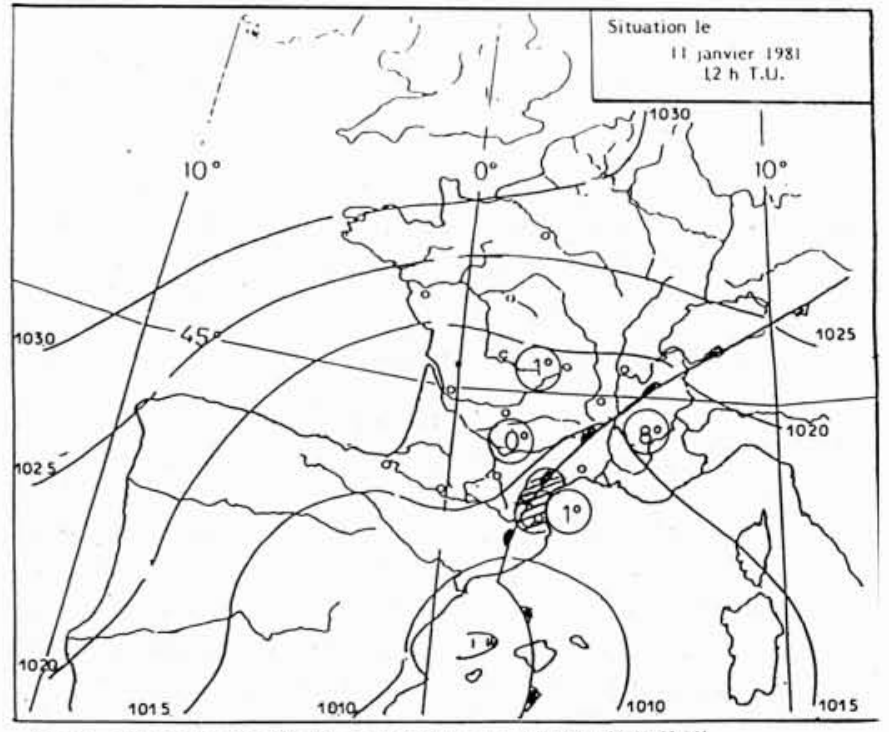

Avaries - Transport : $2 \times 400 \mathrm{kV}-2 \times 225 \mathrm{kV}-1 \times 150 \mathrm{kV}-14 \times 63 \mathrm{kV}$ Distribution : $\mathrm{CD}$. Carcassonne - Perpignan

Observations: manchons de $16 \mathrm{~cm}$ (agent du CD. Carcassonne)

Observations météorologiques à Perpignan : Précipitations à Mas Nérel

\begin{tabular}{|c|c|c|c|c|c|c|c|c|c|c|c|c|c|c|}
\hline \multirow{2}{*}{\multicolumn{2}{|c|}{$\begin{array}{l}\text { Heures } \\
\text { Températures }\end{array}$}} & 00 & 03 & 06 & 09 & 12 & 15 & 18 & 21 & 00 & 03 & 06 & & \\
\hline & & 4.5 & 4.2 & 4.1 & 4.1 & 4.0 & 1. & .5 & 1.1 & 1.3 & 1.5 & 2. & & \\
\hline \multirow{2}{*}{$\ddot{z}$} & Direction & 34 & 34 & 32 & 32 & 30 & 33 & 34 & 33 & 33 & 33 & 34 & & \\
\hline & Force $\mathrm{m} / \mathrm{s}$ & 5 & 3 & 4 & 2 & 4 & 3 & 1 & 4 & 5 & 5 & 2 & & \\
\hline \multicolumn{2}{|c|}{ Précipitations $\mathrm{mm}$} & & & & & 3. & & 38. & & 40. & & 13. & & \\
\hline \multicolumn{2}{|c|}{ Chronologie } & & & & & & - & & & & iy & & & \\
\hline
\end{tabular}

NB. Inversion de températures entre sol et $500 \mathrm{~m}$

Figure 5 


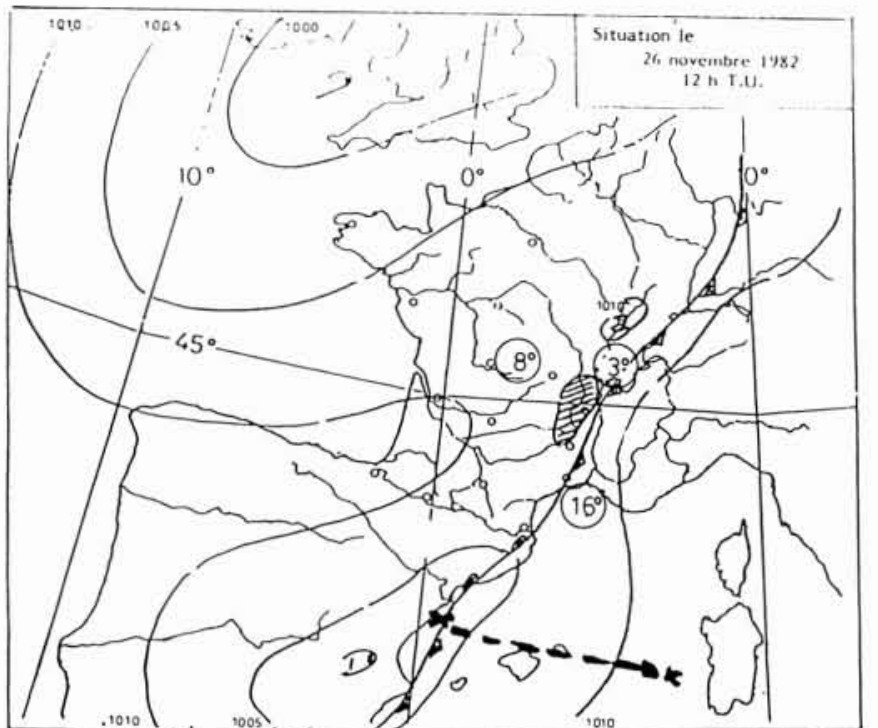

Avaries - Transport : $1 \times 400 \mathrm{kV}-8 \times 225 \mathrm{kV}-1 \times 150 \mathrm{kV}-50 \times 63 \mathrm{kV}$ C.D. St-Etienne - Valence - Le Puy

Observations : manchons 15 à $20 \mathrm{~cm}$ d'après équipes Distribution. Incident débutant vers $20 \mathrm{~h}$ se prolongeant dans la nuit.

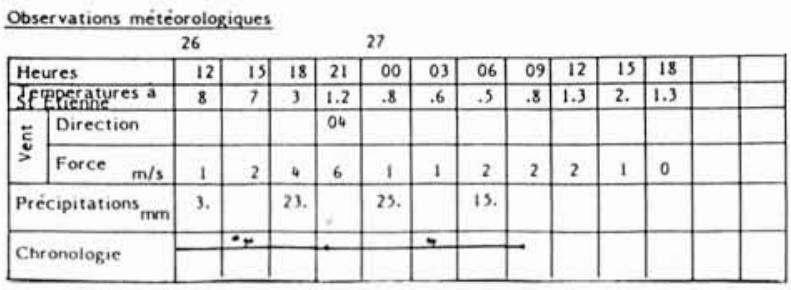

NB. is St-Etienne vent calme a partir de $19 \mathrm{~h}$ ie 26. jusqu'a $11 \mathrm{~h}$ le 27 Rs. Lyon $12 \mathrm{~h}$ : bothermie $=0^{*}$ sur $2000 \mathrm{~m}$

\section{Figure 6}

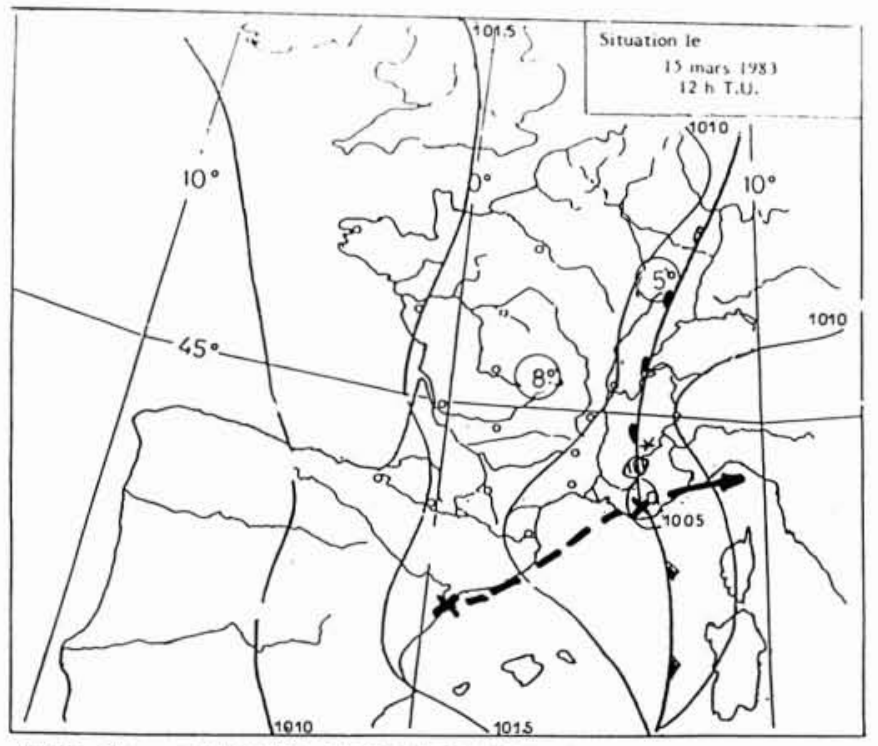

Avaries - Transport $2 \times 225 \mathrm{kV}=1 \times 150 \mathrm{kV}=4 \times 63 \mathrm{kV}$ Distribution C.D. Gap partiel

Altitude : $1000-1100$

Premiers incidents vers $17 \mathrm{~h}$ et sur:out à partir de $20 \mathrm{~h}$.

Observations météorologiques à Embrun

\begin{tabular}{|c|c|c|c|c|c|c|c|c|c|c|}
\hline \multicolumn{2}{|c|}{\begin{tabular}{|l} 
Heures \\
\end{tabular}} & 15 & 09 & 12 & 15 & 18 & 21 & $\frac{16}{00}$ & 03 & 06 \\
\hline \multicolumn{2}{|c|}{ Températures } & 3.5 & 2.8 & 2.8 & .6 & .6 & 2.3 & 3.0 & .5 & -.7 \\
\hline \multirow{2}{*}{$\ddot{\xi}$} & Direction & 04 & 04 & 04 & 04 & 04 & 04 & 04 & 20 & 0 \\
\hline & Force $\mathrm{m} / \mathrm{s}$ & 8 & 9 & 9 & 4 & s & 9 & 5 & 2 & 0 \\
\hline \multicolumn{2}{|c|}{$\frac{1}{\text { Precipitations }}$} & .8 & & & & 20.9 & & & & 3.0 \\
\hline \multicolumn{2}{|c|}{\begin{tabular}{|l|} 
Chronologie \\
\end{tabular}} & $0710^{-}$ & & 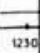 & & & & & & \\
\hline
\end{tabular}

N3. a I'ouest de Serre Poncon precipitations entre 40 et $50 \mathrm{~mm}$ en $12 \mathrm{~h}$.
Manitestations orageuses (foudre).
D'après l'expérience des électriciens japonais, les bagues sont efficaces contre les manchons de neige type " dépression ", très mouillée, et qui tournent en glissant sur la surface du câble. En cas de neige type "mousson " relativement sèche et légère, avec peu de vent, qui se dépose en coiffant progressivement le câble, mais sans beaucoup tourner, elles ont par contre peu d'effet (si ce n'est peut-être qu'elles fractionnent le manchon avant sa chute, évitant ainsi le coup de fouet).

On peut donc logiquement penser que les bagues auraient évité les avaries de Cherbourg (février 1970), Vallée du Rhône (mars 1974) et du Languedoc Roussillon (janvier 1981). A Saint-Etienne (novembre 1982) la neige était aussi très mouillée $(\rho=0,14$ au sol ce qui est vraiment lourd pour la neige fraîche 0,30 sur le câble) mais vu la quasi-absence du vent, on peut se demander si le mouvement de rotation autour des câbles aurait été suffisant pour que les bagues agissent. Pourtant le vent du Nord-Est de 4 à $6 \mathrm{~m} / \mathrm{s}$ enregistré à Bron de $18 \mathrm{~h}$ à $21 \mathrm{~h}$ le 26 , laisse penser qu'il y avait aussi du vent qui remontait les vallées de Giers et de la Brévenne, entre l'Arbresle et Tarare.

\subsection{Evolution des risques de neige collante avec l'alti- tude.}

Parmi les incidents présents au $\S 2.3$ figure le cas du 15 mars 83 qui a affecté dans la région d'Embrun une tranche d'altitude $1000-1100 \mathrm{~m}$. Les incidents étendus à cette altitude sont rares, peut être parce que les lignes y sont plus résistantes.

On peut néanmoins se demander, sans nier les risques en altitude, au vu des incidents récents, si la neige collante n'est pas, dans sa forme la plus grave, un phénomène de basses couches (idée partagée par Y. Sakamoto).

Sur un échantillon de 66 incidents on relève :

- 13 incidents en-dessous de $300 \mathrm{~m}$

- 29 incidents entre 300 et $700 \mathrm{~m}$

- 7 incidents entre 700 et $1000 \mathrm{~m}$

- 13 incidents au-dessus de $1000 \mathrm{~m}$ (Alpes, Pyrénées, souvent ponctuels).

Les $2 / 3$ des incidents, dont tous les cas graves, ont eu lieu an-dessous de $700 \mathrm{~m}$; on peut essayer d'expliquer ce fait par la diminution des chutes de neige par températures positives avec l'altitude ( $90 \%$ à Montpellier) $50 \%$ à Milhau $(700 \mathrm{~m}) 10 \%$ à l'Aigoual $(1500 \mathrm{~m})$, même si ces pourcentages ne concernent pas des échantillons de même taille; l'éloignement de la mer est peut être un autre facteur explicatif.

3 - Cartographie des risques de neige collante

Ainsi que nous l'avons indiqué précédemment les risques de neige collante sont étroitement liés à l'intensité des précipitations. Le gradex, que nous utilisons en hydrologie depuis une vingtaine d'années, est le paramètre qui résume le plus efficacement la relation intensité-fréquence au voisinage et au-delà de la valeur décennale. 


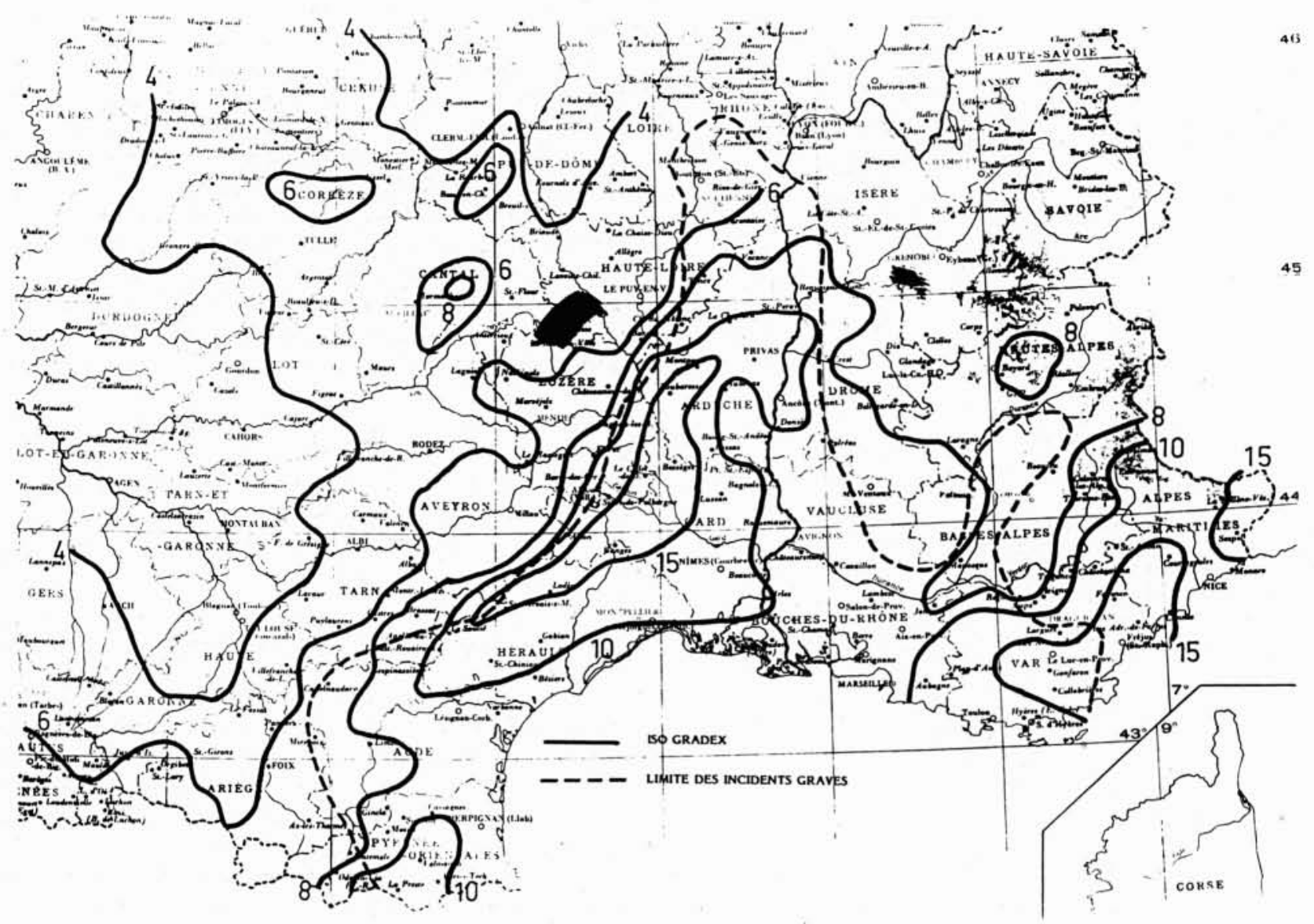

Figure 8 - Iso gradex des précipitations en 6 heures (novembre-mars).

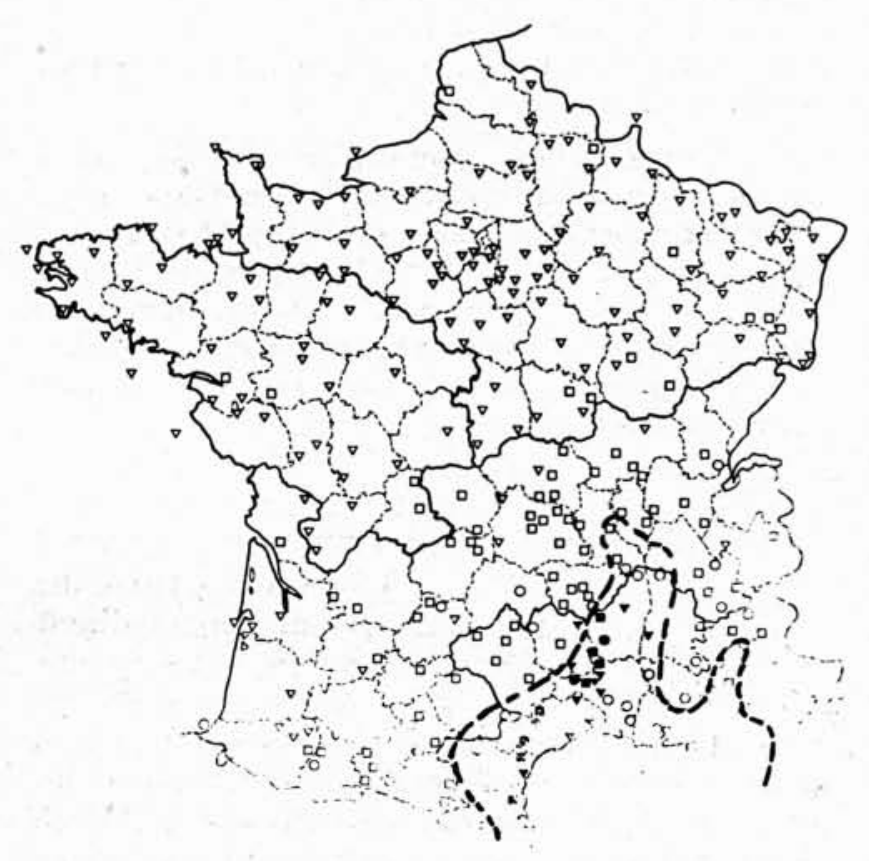

Figure 9 - Gradex des pluies maximales locales de 1 jour (extrait de la publication: Analyse des pluies de 1 à 10 jours, Bureau de l'eau, CEMAGREF, juillet 1979).

$$
\begin{aligned}
& \text { Échelles: } \nabla 4 \text { à } 8 \mathrm{~mm} \text { (in- } \\
& \text { clus) } \\
& \text { ㅁ } 8 \text { à } 12 \mathrm{~mm} \\
& \text { O } 12 \text { à } 17 \mathrm{~mm} \\
& \text { v } 17 \text { à } 25 \mathrm{~mm} \\
& \text { - } 25 \text { à } 35 \mathrm{~mm} \\
& \text { - supérieur à } 35 \\
& \mathrm{~mm}
\end{aligned}
$$




\subsection{Cartographie sur le Sud de la France}

Pour effectuer les études demandées par les C.R.T.T. Sud-Est et Sud-Ouest, nous avons été amenés à calculer les gradex des précipitations en 6 heures des mois d'hiver novembre à mars, pour environ 800 stations climatologiques.

La figure 8 représente les Iso Gradex déduits de ces informations, nous y avons reporté des incidents graves depuis 1970 .

On constate que la limite de la zone des incidents englobe les régions à fort gradex, à l'exception de l'arrière pays niçois. Mais on sait qu'il y a eu aussi de fréquents incidents sur la ligne alimentant Isola 2000. On peut en première appoximation considérer comme zone à risque de neige collante, celle qui est limiée par l'Iso Gradex $8 \mathrm{~mm}$ de la précipitation en 6 heures.

A l'intérieur de cette zone l'évolution du gradex avec l'altitude, ne doit pas être interprétée comme un accroissement similaire des risques, qui ne sont sans doute qu'un peu supérieurs le long des contreforts cévenols, à ce qu'ils sont dans les plaines côtières du Languedoc.

\subsection{Extension à l'ensemble de la France}

L'étude publiée par le Bureau de l'Eau et le C.E.M.A.G.R.E.F. en 1979, concernant une sélection de 300 postes climatologiques présente en conclusion, une carte des gradex de la précipitation journalière.

Bien que cette carte se réfère aux précipitations journalières maximales de toute l'année, elle peut être considérée comme une première ébauche de ce que nous cherchons. On y retrouve naturellement le risque, maximal près de la Méditerranée (Gradex $>17$ ) et beaucoup plus faible sur la moitié Ouest de la France.

Nous avons donc entrepris de préciser le tracé de cette carte, en la limitant à la période novembre-mars, et en tenant compte des variations régionales du rapport Gradex $6 \mathrm{~h} /$ Gradex 24 heures en hiver.

\section{Conclusion}

Aussi surprenant que cela paraisse, les électriciens de réseau ne disposent pas à l'heure actuelle en France, d'un dispositif opérationnel qui leur permette d'enregistrer les forces, horizontales et verticales, qui s'exercent sur les supports des lignes en service. Tant qu'on se contentera de quelques estimations douteuses des surcharges, supputées à l'occasion de quelques avaries exceptionnelles, il est vain d'espérer ajuster les coefficients d'un modèle d'accrétion.

Néanmoins, la question urgente qui nous est posée est de délimiter les régions à fort risque, où les protections (pose de bagues et d'antitorsion, espaceurs, chauffage) sont à installer en priorité, pour éviter la solution ruineuse que serait un renforcement généralisé des supports, indistinctement sur toute la France - L'interprétation de notre fichier des avaries françaises, et l'étude de leurs circonstances météorologiques, étayée par la précieuse expérience des électriciens japonais, permettent de com- prendre au moins les grandes lignes du mécanisme physique de la neige collante : nous avons pu en déduire que le premier facteur discriminant entre régions est l'intensité des précipitations orageuses d'hiver - l'extension à l'ensemble de la France des cartes du gradex, que nous avions déjà ébauchée à la demande des C.R.T.T. de Marseille et Toulouse, est en cours, nouvelle contribution, inattendue, de l'hydrologie à la "pénétration de l'électricité ". -

Références bibliographiques

\section{Amérique du nord :}

[1] G.A. Mac KAY, H.A. THOMPSON. - Estimating the Hazard of Ice Accretion in Canada from climatological Data. Journal of Applied Meteorology, 8-6 - 1969.

[2] P.M. CHAINE. - Variabilité du Verglas au Québec. Etude Ministère de l'Environnement, 29 avril 1973.

[3] D. BAUER. - Snow accretion on power lines. Atmosphere, Vol. $11 \mathrm{n}^{\circ} 3,1973$.

[4] P.M. Chaine, R.W. Verge, G. CAStonguay, J. GARIEPY. - Chargement dû au vent et au verglas au Québec. Etude II, 29 avril 1973.

[5] P.M. CHAINE, P. SKEATES. - Sélection des critères de la charge due à la glace et au vent - Etude III, 9 octobre 1974.

[6] P.M. CHAINE, G. CASTONGUAY. - Une nouvelle approche de la notion d'épaisseur radiale de la glace appliquée $\dot{a}$ des conducteurs du type en faisceaux. Etude IV, Toronto 1974.

[7] P.M. CHAINE. - Givrage dans les nuages. Etude V, Toronto.

[8] B. FELIN. - The observation of Rime and Glaze deposits in Québec. Hydro-Québec, mars 1976.

[9] W. BENDEL \& D. PATON. - A review of the effect of Ice storms on the power Industry. Journal of Applied Meteorology, déc. 81.

[10] U.S. ARMY, CRREL. - Atmospheric Icing of structures (C.R. du séminaire tenu à Hanover les 1.3 juin 1982). CRREL - Special Report 83-17.

\section{Japon :}

[11] SHODA M. - Studies on snow accretion. Studies on Snow and Ice, $\mathrm{n}^{\circ} \mathrm{I}$ pp. 50-72 (1953), Japon.

[12] T. NAKANO \& al. HOKKAIDO \& FURUKAWA EPC. Collapse of Towers due to snow accumulation and its prevention by making conductor - snow resistant CIGRE, 1974.

[13] T. MINYU \& al. FURUKAWA EPC. - Development of snow-resistant conductors CIGRE, 1981, symposium de Stockolm.

[14] D. KUROIWA, G. WAKAhAMA, K. Goto. - Snow accretion on electric wires and its prevention. Journal of Glaciology, Vol. 19, $\mathrm{n}^{\circ} 81$, pp. 479-487 (1977).

[15] G. WAKAHAMA. - Experimental studies of snow accretion on electric lines developped in a strong wind. Natural Disaster Science, Vol. 1, n 1, pp. 21-33 (1979).

[16] Institute of Electrical Engineers of Japan. - Design standards on structures for transmission (Translation of an Excerpt of JEC $127-(1979)$.

[17] Y. SAKAMOTO (CRIEPI). - Pecularities of the Present Snow Accretion (1981).

[18] Federation of Electric Power Companies. - Snow Damage to Transmission and Distribution Lines in Japan of the Tohoku Electric Power Co and Hokuriku Electric Power Co (juin 1981) - (Description des incidents de Sendai 24.12.80 et Toyama 3.1.81). 
19] Y. SAKAMOTO. - Methods of estimating the Amount of Snow accretion on Electric Wire (Methods developped hitherto and their evaluation - 1981).

20] Y. SAKAMOTO \& Y. HIRAYAMA. - Méthode d'estimation de la charge de neige collante sur les lignes aériennes Rapport $n^{\circ} 181519$ du CRIEPI (mars 1982 en Japonais).

\section{France :}

[21] P. GUILLOT. - Commentaire sur l'épisode de neige collante du 24. 25 décembre 1957 en Lozère et Haute-Loire reproduit par R. JALU dans La Météorologie, janv.-mars 1958, pp. $69-73$.

[22] C.R.T.T.N. S/Gr CAEN. - Rapports divers sur incident Cotentin, (Mars 1970).

[23] V. DENEAU, D. DUBAND. - Formation de neige collante sur les lignes de transport: possibilité de prévision, (note D.T.G. du 30 octobre 1973 et du 25 janvier 1974).

[24] Direction du Transport. - Analyses des avaries dues aux surcharges de givre ou de neige. (C.R. d'un groupe de travail - 1973).

[25] C.E.R.T. - Direction Études Appliquées Lignes. - Analyse des avaries survenues dans le Sud-Est, les 3 et 4 mars 1974.

[26] V. DENEAU. - Neige collante. Note sur la répartition des incidents). Note D.T.G. du 10 septembre 1975).

[27] C. PLANCHER. - Évaluation du risque de neige collante sur les lignes du Sud-Est. (Note D.T.G. du 26 mai 1976).

[28] C. PLANCHER. - Etudes de quelques cas d'avaries. (Note D.T.G. du 6 août 1976).
[29] G. DUFOUR. - La neige collante. (Thèse de fin d'Études d'Ingénieur des Travaux de la Météo. Rapport de stage à E.D.F. - D.T.G. Lyon), juin 1976.

[30] D.T.G. - Fiche d'information $n^{\circ} 61$ sur la neige collante sur les conducteurs aériens (janvier 1977).

[31] V. DENEAU. - Evaluation du risque de neige collante sur les lignes de transport du Sud-Ouest. (Note D.T.G. du 23 novembre 1977).

[32] C.D. MONTPELLIER, Carcassonne. - Cartes de givre.

[33] C.E.R.T. - Avaries neige janvier 1981 : hypothèses de calcul, tenue des ouvrages.

[34] C.R.T.T.S.O. - Avaries neige du 11 janvier 1981.

[35] V. DENEAU. - L'accumulation de neige sur les lignes électriques. Les incidents du 11 janvier 1981 (19 octobre 1981).

[36] V. DENEAU. - Givre, neige et réseaux électriques. (Rapport interne 1982).

[37] D. DUBAND \& V. DENEAU. - Prévision journalière de neige collante et de givre. (E.D.F. - D.T.G. - 5 août 1982).

[38] F. FERLAY. - Analyse de l'incident du 26 novembre 1982. E.D.F. - C.R.T.T. ALPES, 16 février 1983.

[39] B. ROSSE, G. VIOLLAZ. - Chute de neige sur le Lyonnais, la Haute Loire avec phénomène de neige collante (26 novembre 1982). (E.D.F. - D.T.G. LYON, mars 1983);

[40] V. DENEAU. - Neige collante - Bilan et perspectives. (Rapport interne 1983).

[41] P. GUILLOT. - Mission neige collante au Japon en octobre 82. Eléments du rapport de mission et quelques réflexions. S.H.F. - Section de Glaciologie (21-22 mars 1983).
$M$. le Président remercie M. DENEAU et ouvre la discussion.

M. MARBOUTY constate que le problème n'a été posé qu'en fonction des dégâts occasionnés, ce qui conduit à n'examiner que les températures comprises entre 0 et $3^{\circ} \mathrm{C}$. Il souligne la possibilité de formation de manchons de $15 \mathrm{~cm}$ de diamètre, par des températures négatives, avec de la neige de densité inférieure à 0,2 . Mais ces manchons n'occasionnent peut-être pas de dégâts. D'autre part les conditions d'isothermie envisagées sont plutôt une conséquence qu'une condition, le paramètre important est l'intensité de la précipitation.

Pour $M$. DENEAU l'échantillonnage est effectivement basé sur les incidents recensés, il se peut qu'on ait ainsi laissé passer des cas de neige collante. Quant à l'isothermie elle dépend des cas et il décrit la neige collante de 1982 en Languedoc où une baisse brutale de température de $3{ }^{\circ} \mathrm{C}$ a été observée, avec des vents qui dans tous les cas venaient de l'intérieur.

$M$. le Président pose la question des conditions d'existence des manchons.

M. DENEAU présente alors les travaux japonais sur l'efficacité des bagues. Il semble qu'il se forme moins de manchons mais cela suppose qu'il y ait un minimum de vent. Ce qui était le cas en 1974 ainsi qu'en 81 en Languedoc. Par contre le cas de St Etienne pose une interrogation étant donné l'absence du vent.

M. AUGUSTIN s'interroge sur les dégâts causés ailleurs que sur les lignes électriques et pose la question de savoir ce qui se passe sur la végétation.

$M$. DENEAU répond qu'il ne s'est intéressé qu'au phénomène électrique, en essayant de rentrer aussi en contact avec les PTT, qui eux aussi ont des problèmes, surtout avec les poteaux qui cassent. Quant aux arbres le problème est différent car la rotation ne se produit pas.
M. DALLE fait état du voyage d'étude effectué au Japon au Centre d'Ishiuchi près de Tokyo, où des expériences ont été réalisées entre -2 et $+4^{\circ} \mathrm{C}$. Mais les dépouillements ne seront disponibles que dans quelques mois.

M. ADMIRAT commente la question de M. MARBouty en faisant une distinction entre l'accrétion et le phénomène de collage. Alors qu'au début pour qu'il y ait collage il faut qu'il se forme un film d'eau capillaire. Une fois la neige déposée on revient à un contact neige/neige et la construction du manchon se poursuit. Par température négative il y a eu des accrétions de plus de $60 \mathrm{~cm}$ de densité faible. Par contre par température positive la densité est très élevée. D'autre part il remarque l'importance de la région du Sud-Est dans ces phénomènes avec des poussées du Sud et pose la question de l'existence de telles situations aussi bien en été qu'en hiver.

Selon $M$. BLIN cette partie de la Méditerranée est un chaudron. Que ce soit l'air chaud saharien ou l'air de l'Atlantique ils s'humidifient beaucoup en passant sur la Méditerranée. Au contact du relief les précipitations sont abondantes que ce soit en hiver ou en été. De plus il y a une forte instabilité convective.

En effet souligne $M$. DENEAU dans la plupart des cas de neige collante il $\mathrm{y}$ a des phénomènes orageux. De plus les jours précédents on a constaté un refroidissement des reliefs avoisinants.

$M$. le Président demande si le phénomène de collage ne débute pas la nuit.

M. DENEAU précise qu'il n'y a pas d'observation nette en ce sens mais qu'il s'écoule au moins 6 heures avant que les ennuis se manifestent. 